Preprint typeset in JHEP style - HYPER VERSION

hep-th/0304081

\title{
Physical wavelets: Lorentz covariant, singularity-free, finite energy, zero action, localized solutions to the wave equation
}

\author{
Matt Visser* \\ School of Mathematical and Computing Sciences, Victoria University of Wellington, \\ New Zealand. \\ E-mail: matt.visser@vuw.ac.nz
}

\begin{abstract}
Particle physics has for some time made extensive use of extended field configuations such as solitons, instantons, and sphalerons. However, no direct use has yet been made of the quite extensive literature on "localized wave" configurations developed by the engineering, optics, and mathematics communities. In this article I will exhibit a particularly simple "physical wavelet" - it is a Lorentz covariant classical field configuration that lives in physical Minkowski space. The field is everwhere finite and nonsingular, and has quadratic falloff in both space and time. The total energy is finite, the total action is zero, and the field configuration solves the wave equation. These physical wavelets can be constructed for both complex and real scalar fields, and can be extended to the Maxwell and Yang-Mills fields in a straightforward manner. Since these wavelets are finite energy, they are guaranteed to be classically present at finite temperature; since they are zero action, they can contribute to the quantum mechanical path integral at zero "cost".
\end{abstract}

KEYWORDS: Wavelet, localized wave, soliton, instanton, sphaleron.

\footnotetext{
* Research supported by the Marsden Fund administered by the Royal Society of New Zealand
} 


\section{Contents}

1. Motivation 1

2. Complex scalar field 2

3. Real scalar field 5

4. Maxwell field

5. Yang-Mills field 9

6. Null $\zeta$

7. Spacelike $\zeta \quad 10$

8. Discussion 10

\section{Motivation}

While the particle physics community has for some time made extensive use of extended field configurations such as solitons, instantons, and sphalerons, no direct use has yet been made of the quite extensive literature on "localized wave" configurations developed by the engineering, optics, and mathematics communities. (For selected references see [1, 2, 3, 4, 5. 6. .) These localized waves are classical solutions of the wave equation that are partially localized in space or time, this localization generally coming at a cost such as infinite total energy and/or instability (leading to dispersion or diffraction). The catalogue of known localized waves is large and growing []], but most of the known examples are not in a form that would be easy to apply to particle physics problems.

In this article I will exhibit a particularly simple "physical wavelet" that is more promising from a particle physics standpoint. It satisfies the properties that:

- It is a localized wave that solves the wave equation.

- It is a Lorentz covariant classical field configuration that lives in physical Minkowski space.

- The field is everywhere finite and nonsingular, and has quadratic falloff in both space and time.

- The total energy is finite, depending on the peak field and the width of the pulse. 
- The total action is zero.

These physical wavelets can be constructed for both complex and real scalar fields. Extending these idea to the Maxwell and Yang-Mills fields is straightforward. The simplest case is that of the complex scalar field and it is to that case that I first turn.

\section{Complex scalar field}

Let $\eta_{a b}=\operatorname{diag}[+1,-1,-1,-1]$ be the Minkowski metric [particle physics signature], let $x_{0}$ be an arbitrary 4 -vector, and let $\zeta^{a}$ be arbitrary timelike 4 -vector, then

$$
\phi(x)=-\frac{\phi_{0}\left(\eta_{a b} \zeta^{a} \zeta^{b}\right)}{\eta_{a b}\left[x^{a}-x_{0}^{a}-i \zeta^{a}\right]\left[x^{b}-x_{0}^{b}-i \zeta^{b}\right]}
$$

is a Lorentz covariant, finite energy, zero-action solution of the d'Alembertian wave equation $\Delta \phi=0$. The "center" of the pulse is at $x_{0}$ and its "width" is $a=\sqrt{\eta_{a b} \zeta^{a} \zeta^{b}}$. The field is everywhere finite and in fact

$$
|\phi(x)| \leq\left|\phi_{0}\right|
$$

To see this, use the fact that $\zeta$ is timelike. Then, using the manifest Lorentz covariance of the field configuration, we can without loss of generality first translate $x_{0} \rightarrow 0$, and then go into the zero-momentum frame where

$$
\zeta^{a}=(a, 0,0,0)
$$

Then the field configuration is

$$
\phi(x)=-\frac{\phi_{0} a^{2}}{[t-i a]^{2}-x^{2}-y^{2}-z^{2}} .
$$

That is

$$
\phi(x)=\frac{\phi_{0} a^{2}}{r^{2}-t^{2}+a^{2}+2 i a t} .
$$

Once written in this form it is a straightforward exercise to verify that the wave equation is satisfied. To see that the field is everywhere bounded note

$$
\begin{aligned}
|\phi|^{2} & =\frac{\left|\phi_{0}\right|^{2} a^{4}}{\left(r^{2}-t^{2}+a^{2}\right)^{2}+4 a^{2} t^{2}}=\frac{\left|\phi_{0}\right|^{2} a^{4}}{\left(r^{2}+t^{2}+a^{2}\right)^{2}-4 r^{2} t^{2}} \leq \\
& \leq \frac{\left|\phi_{0}\right|^{2} a^{4}}{\left(r^{2}+t^{2}+a^{2}\right)^{2}-\left(r^{2}+t^{2}\right)^{2}}=\frac{\left|\phi_{0}\right|^{2} a^{4}}{a^{4}+2 a^{2}\left(r^{2}+t^{2}\right)} \leq\left|\phi_{0}\right|^{2} .
\end{aligned}
$$

From the penultimate inequality we also derive

$$
|\phi|^{2} \leq \frac{1}{2}\left|\phi_{0}\right|^{2} \frac{a^{2}}{r^{2}+t^{2}}
$$

demonstrating the promised quadratic falloff in both space and time. Indeed for fixed $t$ the magnitude of the field is maximized when

$$
r^{2}=\max \left\{t^{2}-a^{2}, 0\right\},
$$


showing that the configuration disperses to spatial infinity at both $t \rightarrow \pm \infty$.

To calculate the 4-momentum, we remind the reader that the stress-energy tensor for a massless complex scalar is

$$
T_{a b}=\frac{1}{2}\left[\phi_{a}^{*} \phi_{b}+\phi_{a} \phi_{b}^{*}\right]-\frac{1}{2} \eta_{a b}|\nabla \phi|^{2} .
$$

Then

$$
\begin{aligned}
\nabla_{a} T^{a b} \equiv & \frac{1}{2} \Delta \phi^{*} \nabla_{b} \phi+\frac{1}{2} \nabla^{a} \phi^{*} \nabla_{a} \nabla_{b} \phi+\frac{1}{2} \Delta \phi \nabla_{b} \phi^{*}+\frac{1}{2} \nabla^{a} \phi \nabla_{a} \nabla_{b} \phi^{*} \\
-\frac{1}{2} \nabla^{b} \phi^{*} \nabla_{a} \nabla_{b} \phi^{*}-\frac{1}{2} \nabla^{a} \phi^{*} \nabla_{a} \nabla_{b} \phi & \\
\equiv & \frac{1}{2}\left[\Delta \phi^{*} \nabla_{b} \phi+\Delta \phi \nabla_{b} \phi^{*}\right],
\end{aligned}
$$

which vanishes by the equations of motion. But this means that

$$
P^{\mu}=\oint T^{a b} \mathrm{~d} \Sigma_{b}
$$

is a conserved quantity, the 4-momentum of the configuration, which is independent of the particular spacelike hypersurface $\Sigma$ chosen to do the integration. By simple dimensional analysis

$$
P^{a}=C\left|\phi_{0}\right|^{2} \zeta^{a}
$$

where $C$ is a dimensionless number to be calculated. [Note that $\zeta^{a}$ has the dimensions of a position vector - a distance.] The energy density is

$$
\rho=\frac{1}{2}\left[\left|\partial_{t} \phi\right|^{2}+\left|\partial_{r} \phi\right|^{2}\right]
$$

and in the zero-momentum frame is easily calculated to be

$$
\rho=\frac{2 a^{4}\left|\phi_{0}\right|^{2}\left(r^{2}+t^{2}+a^{2}\right)}{\left(r^{2}-t^{2}+a^{2}+2 i a t\right)^{2}\left(r^{2}-t^{2}+a^{2}-2 i a t\right)^{2}} .
$$

For arbitrary $t$ this integrates to

$$
\mathcal{E}=\oint \mathrm{d}^{3} r \rho=\int_{0}^{\infty} 4 \pi r^{2} \rho=\frac{1}{2} \pi^{2}\left|\phi_{0}\right|^{2} a
$$

(This is independent of $t$ as it should be.) This is the invariant mass of the field configuration. By spherical symmetry, the total momentum is zero. Thus, for any timelike $\zeta^{a}$

$$
P^{a}=\frac{1}{2} \pi^{2}\left|\phi_{0}\right|^{2} \zeta^{a}
$$

Furthermore the Lagrangian is

$$
\mathcal{L}=\frac{1}{2}\left[\left|\partial_{t} \phi\right|^{2}-\left|\partial_{r} \phi\right|^{2}\right]
$$


which evaluates (in the zero-momentum frame) to

$$
\mathcal{L}=\frac{2 a^{4}\left|\phi_{0}\right|^{2}\left(t^{2}+a^{2}-r^{2}\right)}{\left(r^{2}-t^{2}+a^{2}+2 i a t\right)^{2}\left(r^{2}-t^{2}+a^{2}-2 i a t\right)^{2}} .
$$

It is easy to check that

$$
\oint \mathrm{d}^{4} x \mathcal{L}=0
$$

so that the configuration is zero action.

In summary, what we have is a Lorentz covariant, singularity-free, finite energy, zero action, exact localized solution to the d'Alembertian equation. In many ways this configuration has more right to be called an "instanton" than do the instantons of QFT; those instantons live in Euclidean signature. This field configuration lives in real physical time.

Now the fact that there are finite energy solutions to the wave equation is not a surprise; that these finite energy solutions can coalesce, bounce, and disperse without producing field singularities is more interesting. One way of guessing that the field configuration in equation (2.1) is worth investigating is the following: It is easy to convince oneself that in 4 Euclidean dimensions the solution to Laplace's equation with a delta function source at the origin is

$$
\phi(x) \propto \frac{1}{x^{2}+y^{2}+z^{2}+t^{2}} .
$$

Thus in $(3+1)$ Lorentzian dimensions the [singular] solution to the wave equation with a delta function source at the origin is

$$
\phi(x) \propto \frac{1}{x^{2}+y^{2}+z^{2}-t^{2}} .
$$

If the source is now moved to a real position $x_{0}^{a}$ we have

$$
\phi(x) \propto \frac{1}{\left(x-x_{0}\right)^{2}+\left(y-y_{0}\right)^{2}+\left(z-z_{0}\right)^{2}-\left(t-t_{0}\right)^{2}} .
$$

which is still a singular field configuration. Finally, move the source away from physical Minkowski space to the complex position $x_{0}^{a}-i \zeta^{a}$, then

$$
\phi(x) \propto \frac{1}{\left(x-x_{0}+i \zeta^{1}\right)^{2}+\left(y-y_{0}+i \zeta^{2}\right)^{2}+\left(z-z_{0}+i \zeta^{3}\right)^{2}-\left(t-t_{0}+i \zeta^{0}\right)^{2}},
$$

which is essentially equation (2.1) above. This style of approach has been particularly advocated by Kaiser [5]. As we have just seen, if $\zeta$ is timelike the resulting field configuration is singularity free. However, for null and spacelike $\zeta^{a}$, while the field is still a solution of the wave equation, the field is not bounded. Because of the singularities the energy and action integrals then diverge. Details are deferred for now and will be presented in sections 6 and 0 below.

One should also note that in the optics and engineering literature the most commonly used notations are not manifestly Lorentz covariant. Thus it is common to see expressions such as

$$
\phi \propto \frac{1}{x^{2}+y^{2}+\left[b_{1}-i(z+t)\right]\left[b_{2}+i(z-t)\right]}
$$


(see, for instance, [6] ) whose Lorentz transformation properties are less than obvious - in fact this field configuration is equivalent to equation (2.1) with the identification

$$
\zeta^{a}=-\left(\frac{b_{1}+b_{2}}{2}, 0,0, \frac{b_{1}-b_{2}}{2}\right) ; \quad\|\zeta\|=b_{1} b_{2} ;
$$

\section{Real scalar field}

By taking real and imaginary parts of the complex solution above we can write down two solutions for the real scalar field. Namely

$$
\begin{aligned}
\phi_{1} & =\frac{\phi_{0}\left(\eta_{a b} \zeta^{a} \zeta^{b}\right)\left\{\eta_{a b}\left[x^{a}-x_{0}^{a}\right]\left[x^{b}-x_{0}^{b}\right]-\eta_{a b} \zeta^{a} \zeta^{b}\right\}}{\left(\eta_{a b}\left[x^{a}-x_{0}^{a}\right]\left[x^{b}-x_{0}^{b}\right]-\eta_{a b} \zeta^{a} \zeta^{b}\right)^{2}+4\left(\eta_{a b}\left[x^{a}-x_{0}^{a}\right] \zeta^{b}\right)^{2}} ; \\
\phi_{2} & =\frac{2 \phi_{0}\left(\eta_{a b} \zeta^{a} \zeta^{b}\right)\left\{\eta_{a b}\left[x^{a}-x_{0}^{a}\right] \zeta^{b}\right\}}{\left(\eta_{a b}\left[x^{a}-x_{0}^{a}\right]\left[x^{b}-x_{0}^{b}\right]-\eta_{a b} \zeta^{a} \zeta^{b}\right)^{2}+4\left(\eta_{a b}\left[x^{a}-x_{0}^{a}\right] \zeta^{b}\right)^{2}} .
\end{aligned}
$$

As previously, we can without loss of generality translate $x_{0} \rightarrow 0$ and go to the zeromomentum frame $\zeta^{a}=(a, 0,0,0)$, then

$$
\begin{aligned}
& \phi_{1}=\frac{\phi_{0} a^{2}\left\{t^{2}-r^{2}-a^{2}\right\}}{\left(t^{2}-r^{2}-a^{2}\right)^{2}+4 a^{2} t^{2}} ; \\
& \phi_{2}=\frac{\phi_{0} a^{2} 2 a t}{\left(t^{2}-r^{2}-a^{2}\right)^{2}+4 a^{2} t^{2}} .
\end{aligned}
$$

The stress-energy for a real scalar field simplifies

$$
T_{a b}=\phi_{a} \phi_{b}-\frac{1}{2} \eta_{a b}|\nabla \phi|^{2}
$$

Then

$$
\begin{aligned}
\nabla_{a} T^{a b} & \equiv \Delta \phi \nabla_{b} \phi+\nabla^{a} \phi \nabla_{a} \nabla_{b} \phi-\nabla^{b} \phi \nabla_{a} \nabla_{b} \phi \\
& \equiv \Delta \phi \nabla_{b} \phi
\end{aligned}
$$

which vanishes by the equations of motion. The calculation for the energy-momentum 4-vector now yields:

$$
P_{1}^{a}=\frac{1}{4} \pi^{2}\left|\phi_{0}\right|^{2} \zeta^{a}=P_{2}^{a} .
$$

The action integral for both of these field configurations is still zero. $\left[\oint \mathrm{d}^{3} x \mathrm{~d} t \mathcal{L}\left(\phi_{1,2}\right)=0\right.$.]

\section{Maxwell field}

A similar construction can be performed for the Maxwell field. There are a number of choices one could make, and I will simply pick one that leads to a relatively simple field configuration. Start by picking a timelike 4-velocity $V^{a}$ and a spacelike unit vector $m^{a}$ orthogonal to it. Now adopt the ansatz

$$
A^{a}=\left\{V^{a} m^{b}-m^{a} V^{b}\right\} \nabla_{b} \psi .
$$


Here one is automatically in Lorenz gauge [8], $\nabla_{a} A^{a}=0$, and the Maxwell equations reduce to the wave equation $\Delta \psi=0$ for the scalar potential $\psi$. The field configuration is manifestly Lorentz covariant and we can without loss of generality go to the inertial frame where

$$
V^{a}=(1,0,0,0) ; \quad m^{a}=(0 ; \vec{m})
$$

In this inertial frame

$$
A_{a}=(\varphi, \vec{A})=([\vec{m} \cdot \vec{\nabla}] \psi, \vec{m} \dot{\psi}),
$$

where $\vec{m}$ is a constant unit vector. We shall soon see that this is tantamount to working in the zero-momentum frame of the field configuration. The electric field is

$$
\vec{E}=-\vec{\nabla}([\vec{m} \cdot \vec{\nabla}] \psi)+\vec{m} \ddot{\psi}=-\vec{\nabla}([\vec{m} \cdot \vec{\nabla}] \psi)+\vec{m} \nabla^{2} \psi=\vec{\nabla} \times(\vec{\nabla} \times[\vec{m} \psi]),
$$

where we have used the wave equation for $\psi$. The magnetic field is

$$
\vec{B}=\vec{\nabla} \times(\vec{m} \dot{\psi})=-\vec{m} \times \vec{\nabla} \dot{\psi}
$$

The energy density and momentum flux (Poynting vector) are

$$
\rho=\frac{1}{2}\left[\vec{E}^{2}+\vec{B}^{2}\right] ; \quad \vec{S}=\vec{E} \times \vec{B} .
$$

To evaluate total energy and momentum a useful integration by parts (subject to suitable falloff at spatial infinity) is

$$
\begin{aligned}
\oint \mathrm{d}^{3} x\left(\vec{\nabla} \times \vec{X}_{1}\right) \cdot\left(\vec{\nabla} \times \vec{X}_{2}\right) & =\oint \mathrm{d}^{3} x\left(\vec{X}_{1} \times \vec{\nabla}\right) \cdot\left(\vec{\nabla} \times \vec{X}_{2}\right) \\
& =\oint \mathrm{d}^{3} x \vec{X}_{1} \cdot\left[\vec{\nabla} \times\left(\vec{\nabla} \times \vec{X}_{2}\right)\right] \\
& =\oint \mathrm{d}^{3} x \vec{X}_{1} \cdot\left[-\vec{\nabla}^{2} \vec{X}_{2}+\vec{\nabla}\left(\vec{\nabla} \cdot X_{2}\right)\right] \\
& =-\oint \mathrm{d}^{3} x\left\{\vec{X}_{1} \cdot \vec{\nabla}^{2} \vec{X}_{2}+\left(\vec{\nabla} \cdot \vec{X}_{1}\right)\left(\vec{\nabla} \cdot \vec{X}_{2}\right)\right\} .
\end{aligned}
$$

Consequently

$$
\begin{aligned}
\oint \mathrm{d}^{3} x \vec{E}^{2} & =-\oint \mathrm{d}^{3} x\left\{[\vec{\nabla} \times(\vec{m} \psi)] \nabla^{2}[\vec{\nabla} \times(\vec{m} \psi)]\right\} \\
& =+\oint \mathrm{d}^{3} x\left\{(\vec{m} \psi) \nabla^{2} \nabla^{2}(\vec{m} \psi)+(\vec{\nabla} \cdot[\vec{m} \psi]) \nabla^{2}(\vec{\nabla} \cdot[\vec{m} \psi])\right\} \\
& =+\oint \mathrm{d}^{3} x\left\{\psi \nabla^{2} \nabla^{2} \psi+(\vec{m} \cdot \vec{\nabla} \psi) \nabla^{2}(\vec{m} \cdot \vec{\nabla} \psi)\right\} .
\end{aligned}
$$

Now let us assume the potential $\psi$ is spherically symmetric $\psi(r, t)$. Then averaging over angular variables is the same as averaging over orientations of the unit vector $\vec{m}$ and under the angular integral we can effectively replace

$$
m_{i} m_{j} \rightarrow \frac{1}{3} \delta_{i j}
$$


Consequently

$$
\begin{aligned}
\oint \mathrm{d}^{3} x \vec{E}^{2} & =+\oint \mathrm{d}^{3} x\left\{\nabla^{2} \psi \nabla^{2} \psi+\frac{1}{3}(\vec{\nabla} \psi) \cdot \nabla^{2}(\vec{\nabla} \psi)\right\} \\
& =+\oint \mathrm{d}^{3} x\left\{\left(\nabla^{2} \psi\right)^{2}-\frac{1}{3}\left(\nabla^{2} \psi\right)^{2}\right\} \\
& =+\frac{2}{3} \oint \mathrm{d}^{3} x\left\{\left(\nabla^{2} \psi\right)^{2}\right\} .
\end{aligned}
$$

Similarly

$$
\oint \mathrm{d}^{3} x \vec{B}^{2}=-\oint \mathrm{d}^{3} x\left\{(\vec{m} \dot{\psi}) \cdot \nabla^{2}(\vec{m} \dot{\psi})+(\vec{m} \cdot \vec{\nabla} \dot{\psi})(\vec{m} \cdot \vec{\nabla} \dot{\psi})\right\} .
$$

Again invoking spherical symmetry for $\psi$ we have

$$
\begin{aligned}
\oint \mathrm{d}^{3} x \vec{B}^{2} & =-\oint \mathrm{d}^{3} x\left\{\dot{\psi} \nabla^{2} \dot{\psi}+\frac{1}{3}(\vec{\nabla} \dot{\psi}) \cdot(\vec{\nabla} \dot{\psi})\right\} \\
& =+\frac{2}{3} \oint \mathrm{d}^{3} x\{(\vec{\nabla} \dot{\psi}) \cdot(\vec{\nabla} \dot{\psi})\} .
\end{aligned}
$$

Therefore

$$
\mathcal{E}=\oint \mathrm{d}^{3} x \rho=\frac{1}{3} \oint \mathrm{d}^{3} x\left\{\left(\nabla^{2} \psi\right)^{2}+(\vec{\nabla} \dot{\psi}) \cdot(\vec{\nabla} \dot{\psi})\right\}
$$

so the total energy is sensibly positive. Before making further choices regarding the potential $\psi$, consider the total momentum

$$
\vec{\wp}=\oint \mathrm{d}^{3} x \vec{S}=\oint \mathrm{d}^{3} x[\vec{\nabla} \times(\vec{\nabla} \times[\vec{m} \psi])] \times[\vec{\nabla} \times(\vec{m} \dot{\psi})] .
$$

Integration by parts implies

$$
\vec{\wp}=\oint \mathrm{d}^{3} x\left\{(\vec{m} \dot{\psi}) \cdot \nabla^{2}(\vec{\nabla} \times[\vec{m} \psi])\right\}=-\oint \mathrm{d}^{3} x\left\{\dot{\psi} \vec{m} \cdot\left[\vec{m} \times \vec{\nabla}\left(\nabla^{2} \psi\right)\right]\right\}=0,
$$

so that the net momentum is zero. For the action, an integration by parts together with the equations of motion yields

$$
\oint \mathrm{d}^{4} x \frac{1}{2}\left[\vec{E}^{2}-\vec{B}^{2}\right]=\frac{1}{3} \oint \mathrm{d}^{3} x\left\{\left(\nabla^{2} \psi\right)^{2}-(\vec{\nabla} \dot{\psi}) \cdot(\vec{\nabla} \dot{\psi})\right\}=0 .
$$

So we still have a zero action solution.

Up to now the potential $\psi$ has only needed to be spherically symmetric and to satisfy the wave equation (plus some falloff constraint at spatial infinity to allow the integration by parts). The general solution to the wave equation in spherical symmetry is

$$
\psi(r, t)=\frac{1}{r}[f(r+t)-f(r-t)] .
$$

Returning to the energy $\mathcal{E}$ as given in equation (4.21), a further integration by parts, together with the wave equation for $\psi$ yields the computationally convenient form

$$
\mathcal{E}=\frac{1}{3} \oint \mathrm{d}^{3} x\left\{\partial_{t}^{2} \psi \partial_{t}^{2} \psi-\partial_{t} \psi \partial_{t}^{3} \psi\right\} .
$$


Whence

$$
\begin{aligned}
& \mathcal{E}=4 \pi \int_{0}^{\infty} \mathrm{d} r\left\{\left(\partial_{t}^{2}[f(r+t)-f(r-t)]\right)^{2}\right. \\
&\left.-\left(\partial_{t}[f(r+t)-f(r-t)]\right)\left(\partial_{t}^{3}[f(r+t)-f(r-t)]\right)\right\} \\
&=4 \pi \int_{0}^{\infty} \mathrm{d} r\left\{\left(\partial_{r}^{2}[f(r+t)-f(r-t)]\right)^{2}\right.\left.\quad-\left(\partial_{r}[f(r+t)+f(r-t)]\right)\left(\partial_{r}^{3}[f(r+t)+f(r-t)]\right)\right\} \\
&=4 \pi \int_{0}^{\infty} \mathrm{d} r\left\{\left(\partial_{r}^{2}[f(r+t)-f(r-t)]\right)^{2}\right.\left.\quad+\left(\partial_{r}^{2}[f(r+t)+f(r-t)]\right)^{2}\right\} \\
&=8 \pi \int_{0}^{\infty} \mathrm{d} r\left\{\left[\partial_{r}^{2} f(r+t)\right]^{2}+\left[\partial_{r}^{2} f(r-t)\right]^{2}\right\} \\
&=8 \pi \int_{-\infty}^{+\infty} \mathrm{d} s\left[\partial_{s}^{2} f(s)\right]^{2} .
\end{aligned}
$$

Which verifies that the energy is constant in a model-independent manner. Furthermore if $f(s)$ is smooth and satisfies suitable falloff conditions at $s \rightarrow \pm \infty$ then the wavelet will be nonsingular and of finite energy.

To obtain a specific example it only remains to finish the complete specification of the potential $\psi(r, t)$. One particularly simple choice is to take one of the real scalar wavelets of the previous section $\psi \rightarrow \phi_{1,2}$, with $\zeta^{a}=\|\zeta\| V^{a}=a V^{a}$. Since the electric and magnetic fields are now specified in terms of derivatives of a smooth bounded function, the electromagnetic field is similarly smooth and bounded. For the energy, write it in the form

$$
\mathcal{E}=\frac{1}{3} \oint \mathrm{d}^{3} x\left\{\psi \partial_{t}^{4} \psi-\partial_{t} \psi \partial_{t}^{3} \psi\right\}
$$

Whence, for either $\psi \rightarrow \phi_{1,2}$, an integration carried out at arbitrary $t$ yields the timeindependent quantity

$$
\mathcal{E}_{1,2}=\frac{1}{2} \frac{\phi_{0}^{2}}{a}
$$

The calculation has for convenience been carried out in the zero-momentum frame of the wavelet. In a general Lorentz frame we would have

$$
P_{1,2}^{a}=\frac{1}{2} \frac{\phi_{0}^{2}}{a} V^{a}=\frac{1}{2} \frac{\phi_{0}^{2}}{a^{2}} \zeta^{a}
$$

Thus this field configuration, as for the scalar case, is Lorentz covariant, bounded, finite energy and zero action. The vector nature of the Maxwell field has added technical complications, but there is no real change in basic principles.

Aside: In closing this section, I should mention one other wavelet that is particularly attractive. If one makes use of the pseudo-differential operator $\left(\nabla^{2}\right)^{-1 / 2}$ one could write

$$
\psi=\left(\nabla^{2}\right)^{-1 / 2} \phi_{1,2}=\frac{1}{\Gamma(-1 / 2)} \int_{0}^{\infty} \frac{\mathrm{d} t}{t^{3 / 2}} \exp \left[-t \nabla^{2}\right] \phi_{1,2}
$$


With this choice of $\psi$ the energy integral simplifies

$$
\mathcal{E}_{1,2}=\frac{1}{3} \oint \mathrm{d}^{3} x\left\{\left(\partial_{t} \phi_{1,2}\right)^{2}+\left(\nabla \phi_{1,2}\right)^{2}\right\}=\frac{1}{6} \pi^{2}\left|\phi_{0}^{2}\right| a .
$$

The price paid for making the energy look simple is that the electric and magnetic fields are much more complicated to calculate.

Comment: Because the optics and engineering literature generally does not use manifestly Lorentz covariant notation, it can be very time consuming to calculate the 4-momentum of a specific pulse. Indeed only very recently [6] has Lekner provided specific and explicit computations of both $\mathcal{E}$ and $\vec{\wp}$ (as well as the angular momentum) for a pulse similar to that considered above. As expected (once one has the covariant perspective advocated in this article) $\|\vec{\wp}\|<\mathcal{E}$, indicating the existence of a zero-momentum frame for the pulses of this type [6].

\section{Yang-Mills field}

Once we have the Maxwell wavelet above, a Yang-Mills wavelet is straightforward, indeed trivial. Let $\boldsymbol{\Lambda}$ be any constant matrix in the center of the gauge group and set $\mathbf{A}^{a}=A^{a} \boldsymbol{\Lambda}$. The construction is so simple that there is really nothing extra beyond the Maxwell wavelet considered above.

\section{Null $\zeta$}

Let us now return to the original scalar complex wavelet. Suppose the 4-vector $\zeta$ is null. Then because the numerator vanishes identically the original definition above gives $\phi \equiv 0$. We should at a minimum change our field definition to read

$$
\phi(x)=-\frac{\psi_{0}}{\eta_{a b}\left[x^{a}-x_{0}^{a}-i \zeta^{a}\right]\left[x^{b}-x_{0}^{b}-i \zeta^{b}\right]} .
$$

Then without loss of generality we go into the frame

$$
\zeta^{a}=(a, 0,0, a)
$$

and then

$$
\phi(x)=-\frac{\psi_{0}}{[t-i a]^{2}-x^{2}-y^{2}-[z-i a]^{2}} .
$$

That is

$$
\phi(x)=\frac{\psi_{0}}{r^{2}-t^{2}+2 i a(t-z)} .
$$

Note

$$
|\phi(x)|=\frac{\psi_{0}}{\sqrt{\left(r^{2}-t^{2}\right)^{2}+4 a^{2}(t-z)^{2}}} .
$$

The denominator now vanishes when $z-t$ and $x=y=0$, so that the field is divergent on the beam axis. Suppose we write $R=\sqrt{x^{2}+y^{2}}$ then

$$
\phi(x)=\frac{\psi_{0}}{R^{2}+z^{2}-t^{2}+2 i a(t-z)} .
$$


So we see that the field drops off as $1 / R^{2}$ as we move away from the beam axis. [And more critically, the field blows up as $1 / R^{2}$ as we approach the beam axis.] Attempts at calculating the energy and action now lead to divergent integrals. In other words, despite the fact that it still solves the wave equation, for null $\zeta$ this is not a particularly useful field configuration.

\section{Spacelike $\zeta$}

Foe the complex scalar wavelet, suppose the 4 -vector $\zeta$ is spacelike. Then without loss of generality we go into the infinite velocity frame where

$$
\zeta^{a}=(0,0,0, a)
$$

and then

$$
\phi(x)=\frac{\phi_{0} a^{2}}{t^{2}-x^{2}-y^{2}-[z-i a]^{2}} .
$$

That is

$$
\phi(x)=-\frac{\phi_{0} a^{2}}{r^{2}-t^{2}-a^{2}-2 i a z} .
$$

Note

$$
|\phi(x)|=\frac{\phi_{0} a^{2}}{\sqrt{\left(r^{2}-t^{2}-a^{2}\right)^{2}+4 a^{2} z^{2}}} .
$$

The denominator now vanishes when $z=0$ and $x^{2}+y^{2}=a^{2}+t^{2}$. That is, the field is divergent on a time-dependent circle orthogonal to the beam axis. There is a short distance singularity as one approaches this circle, and the energy and action integrals diverge. In other words, despite the fact that it still solves the wave equation, for spacelike $\zeta$ this is not a useful field configuration.

\section{Discussion}

The physical wavelet discussed in this article is important because it represents a qualitatively different extended field configuration of a type not normally encountered in particle physics. The wavelet is neither a soliton, nor an instanton, nor a sphaleron though it shares properties with all three of these extended objects:

- Like the soliton it lives in physical time (Minkowski space, not Euclidean space), and possesses a well-defined 4-velocity.

- Like the instanton it "dies away" in the infinite past and future.

- Like the instanton it possesses a continuously adjustable scale parameter.

- Like the sphaleron it is unstable to dispersal. 
Because the wavelet fields are bounded and finite energy, wavelet configurations will be classically excited at any finite temperature. Because the wavelet configuration has zero action, arbitrarily complicated combinations of these physical wavelets can be added to the field configurations appearing in Feynman's path integral without modifying the phase quantum mechanically there is no "cost" in adding these configurations to the Lorentzian path integral and they will contribute.

Other "localized waves" might be interesting in specific applications but the particular example discussed in this article is important because of its extreme simplicity and pleasant behaviour.

\section{Acknowledgments}

I wish to thank John Lekner for stimulating my interest in these issues. I also wish to thank Damien Martin for bringing the whole Lorenz/Lorentz issue to my attention.

\section{References}

[1] R. W. Ziolkowski, "Exact solutions of the wave equation with complex source locations", J. Math. Phys. 26 (1985) 861-863.

[2] M. K. Tippet and R. W. Ziolkowski, "A bidirectional wave transformation of the cold plasma equations", J. Math. Phys. 32 (1991) 488-492.

[3] J. Lu and J. F. Greenleaf, "Nondiffracting X waves - exact solutions to free-space scalar wave equation and their finite aperture realizations", IEEE Transactions on ultrasonics, ferroelectrics, and frequency control, 39 (1992) 19-31.

[4] J. Lu, H. Zou, and J. F. Greenleaf, "A new approach to obtain limited diffraction beams", IEEE Transactions on ultrasonics, ferroelectrics, and frequency control, 42 (1995) 850-853.

[5] G. Kaiser, "Physical wavelets and their sources: Real physics in complex spacetime," arXiv:math-ph/0303027.

[6] J. Lekner, "Electromagnetic pulses which have a zero momentum frame", submitted to J. Opt. A, 21 November 2002; arXiv:physics/0304022.

[7] Field configurations similar to the one considered in this article may variously be encountered under names such as: "localized waves", "focus wave modes", "pulses", "X-waves", "limited diffraction beams", "wavelets", and "physical wavelets".

[8] The Lorenz gauge was apparently first used by the Danish physicist Ludwig Lorenz (1829-1891), though it is commonly misattributed to the Dutch physicist Hendrik Antoon Lorentz (1853-1928).

L. Lorenz, "On the Identity of the Vibrations of Light with Electrical Currents", Philos. Mag. 34 (1867) 287-301.

J. van Bladel, "Lorenz or Lorentz?", IEEE Antennas Prop. Mag. 33 (1991) 69. 